\title{
Properties of Dwarf Stars in Cygnus OB2
}

\author{
Olga Maryeva ${ }^{1,3}$, S. Yu. Parfenov ${ }^{2}$, M. V. Yushkin ${ }^{1}$, A. S. Shapovalova ${ }^{2}$ and S. Yu. Gorda ${ }^{2}$ \\ ${ }^{1}$ Special Astrophysical Observatory of the Russian Academy of Sciences, Nizhnii Arkhyz, 369167, Russia \\ ${ }^{2}$ Ural Federal University, 51 Lenin Str., Ekaterinburg, 620000, Russia \\ ${ }^{3}$ Email: olga.maryeva@gmail.com
}

(ReCEIVEd August 01, 2015; ACCEPTED December 16, 2015)

\begin{abstract}
We present the results of investigation of five stars, originally classified as dwarfs, belonging to Cyg OB2 association, their stellar, and wind properties. Using both TLUSTY and CMFGEN codes, we derived effective temperatures, surface gravities, chemical abundances, mass-loss rates, and projected rotation velocities. Due to the fact that distance to the stars is well known, we were able to estimate their luminosities. Using evolutionary models, we estimated the ages of these sample stars and find that lower mass ones-MT282 and MT343-belong to older population of the association. Their ages are greater than 10 Myr. The ages of three other stars-MT317, MT299, MT259—are between 4 and 6 Myr.
\end{abstract}

Keywords: stars: atmospheres - stars: early-type - stars: formation - stars: fundamental parameters - stars: individual: Cygnus OB2

\section{INTRODUCTION}

Cygnus OB2 (Cyg OB2), a stellar association discovered by Münch and Morgan in 1953 (Münch \& Morgan 1953), is now one of the leaders in number of massive stars among Galactic OB-associations. According to the estimates of Knödlseder (2000), it includes $2600 \pm 400$ stars and about 100 of them are O-stars located in the $1 \mathrm{deg}^{2}$ region (Comerón et al., 2002; Wright et al. 2010; Wright, Drew, \& Mohr-Smith 2015). Due to it, the central part of association is the region with the highest concentration of hot stars in the Galaxy. The interest of researchers to individual stars and to the association as a whole is not fading. A lot of papers dedicated to the investigation of Cyg OB2 stellar population [like Drew et al. (2008); Comerón \& Pasquali (2012)], estimation of interstellar extinction [like Guarcello et al. (2012)], history of star formation [like Wright et al. $(2014,2015)$ ] have already been published and continue to appear.

Modelling of atmospheres of the stars belonging to an association is an important area of investigation, since it both gives the parameters of individual stars and allows to construct an association Hertzsprung-Russell (H-R) diagram as a whole. The $\mathrm{H}-\mathrm{R}$ diagram in turn provides the information about stellar ages, masses, and history of star formation. Main works devoted to the numerical modelling of stellar atmospheres in Cyg OB2 association are Herrero et al. (1999); Herrero, Puls, \& Najarro (2002); Negueruela et al. (2008). Moreover, individual supergiants were studied by Najarro, Hanson, \& Puls (2011); Maryeva, Klochkova,
\& Chentsov (2013); Maryeva, Zhuchkov, \& Malogolovets (2014).

In the present work, we model the stellar atmospheres of five stars initially assumed to have $\mathrm{V}$ luminosity class (dwarfs). Star \#6 or MT317 ${ }^{1}$ may be called the native resident of association Cyg OB2. It belongs to the first dozen of blue stars noticed by Münch \& Morgan (1953). Based on spatial closeness of these objects, Münch and Morgan have suggested the existence of OB-association in about $2^{\circ}$ around $\gamma$ Cygni. Johnson \& Morgan (1954) classified MT317 as O8 (V). The contemporary researchers (Kiminki et al. 2007; Chentsov et al. 2013) also consider that MT317 is O8 V. Morgan, Meinel, \& Johnson (1954) suggested that star \#16 (MT299) is probably the faint member of the association Cyg OB2. Then, Schulte (1956) included the star \#21 (MT259) into the association. Chentsov et al. (2013) classified MT299 as O7.5 V, while MT259 as B0 V. Massey \& Thompson (1991) also included MT282 and MT343 stars into the association. The latter was subsequently classified by Kiminki et al. (2007) as B1 V, while the first spectrum of MT282 was obtained only recently on the Russian 6-m telescope, and MT282 was classified as B1 IV-V (Maryeva et al. 2016). Among these five stars only the MT259 had previously been studied by Herrero et al. (1999) using stellar atmosphere models.

${ }^{1} \# 6$, \#16, and \#21 are the names of the stars according to the catalogue of Schulte (1956), while MT is the catalogue of Cyg OB2 members by Massey \& Thompson (1991) 
The paper is organised as follows. Observational data and main steps of data reduction are described in the next section. Data analysis and determination of different stellar parameters are in Section 3. The Section 4 presents the results of modelling, while in Section 5, we will discuss the locations of the stars on the H-R diagram and the estimated masses, ages, and mass loss rates. In the Section 6, short conclusions are given. The testing of the method of automatic determination of effective temperature and surface gravity is presented in appendices.

\section{OBSERVATIONAL DATA}

The high resolution spectra of 13 stars in Cyg OB2 had been thoroughly described by Chentsov et al. (2013), who also studied spectral variability of the MT304 (Schulte \#12) hypergiant. In our work, we analysed all three stars MT317, MT299, and MT259 classified by Chentsov et al. (2013) as dwarfs and we used the same spectral data. These data were obtained with the high-resolution Nasmyth Echelle Spectrograph (NES) (Panchuk et al. 2009) on the Russian 6-m telescope. The observations were conducted using the image slicer (Panchuk et al. 2009) and a $2048 \times 2048$ CCD. The extraction of one-dimensional vectors from the twodimensional echelle spectra was performed with the aid of the ECHELLE context of the MIDAS software package, modified by Yushkin \& Klochkova (2005). The removal of the traces of cosmic ray particles was done by means of median filtration of image sequences using standard MIDAs routines. Wavelength calibration was carried out using the spectra of a Th-Ar hollow cathode lamp. Continuum normalisation of spectra was performed manually using DECH software package (Galazutdinov 1996).

Spectra of MT282 and MT343 were also obtained on the Russian 6-m telescope, but with Spectral Camera with Optical Reducer for Photometric and Interferometric Observations (SCORPIO) in the long-slit mode (Afanasiev \& Moiseev 2005). VPHG1200G grism was used providing the spectral range of 4 050-5 $850 \AA \AA$. All SCORPIO spectra were reduced using the $S C O R E$ package ${ }^{2}$. SCORE was written by Maryeva and Abolmasov in IDL language for SCORPIO long-slit data reduction. Package includes all the standard stages of long-slit data reduction process. The final spectra of MT282 and MT343 have spectral resolution $\sim 6 \AA$ (weakly dependent on wavelength) and signal-to-noise ratio $(\mathrm{S} / \mathrm{N})$ per resolution element in continuum near $4900 \AA \sim 50$ and $\sim 70$, correspondingly. The data used in this work is summarised in Table 1.

\section{METHODS OF DATA ANALYSIS}

For estimation of physical parameters of the sample stars, we used two atmospheric modelling codes-TLUSTY (Hubeny \&

\footnotetext{
${ }^{2} \mathrm{http}: / /$ www.sao.ru/hq/ssl/maryeva/score.html
}

PASA, 33, e002 (2016)

doi:10.1017/pasa.2015.53
Lanz 1995; Lanz \& Hubeny 2003) and CMFGEN (Hillier \& Miller 1998).

Effective temperature $T_{\text {eff }}$, surface gravity $\log$ g, and projected rotation velocities $v \sin i$ were estimated using stellar atmosphere models computed with TLUSTY. TLUSTY is the code for computing plane-parallel, horizontally homogeneous model stellar atmospheres in radiative and hydrostatic equilibrium, developed by Hubeny \& Lanz (1995); Lanz \& Hubeny (2003). Departures from local thermodynamic equilibrium (LTE) are allowed for a set of occupation numbers of selected atomic and ionic energy levels. Latest version of the program accounts for a fully consistent, non-LTE metal line blanketing, as well as convection (http://nova.astro.umd.edu/ index.html). For the analysis, we used the grids of TLUSTY stellar atmosphere models and synthetic spectra computed by us and two pre-computed grids. These pre-computed grids are OSTAR2OO2 grid with solar metallicity and microturbulent velocity $v_{\text {turb }}=10 \mathrm{~km} \mathrm{~s}^{-1}$ (Lanz \& Hubeny 2003) and BSTAR2006 grid with solar metallicity and $v_{\text {turb }}=2 \mathrm{~km} \mathrm{~s}^{-1}$ (Lanz \& Hubeny 2007).

For determination of wind parameters, bolometric luminosity and chemical composition we used CMFGEN code (Hillier \& Miller 1998). This code is modelling spherically symmetric extended outflows using the full comoving-frame solution of the radiative transfer equation. CMFGEN incorporates line blanketing, the effects of Auger ionisation and clumping. The level populations are calculated through the rate equations. A super-level approach is used to reduce the scale of the problem (and thus the computing time).

\subsection{Determination of projected rotational velocities}

For estimation of rotational velocities $v \sin i$, we used synthetic spectra from the TLUSTY grids mentioned above. From these grids, we chose the spectrum computed for the effective temperature $T_{\text {eff }}$ and surface gravity $\log g$ corresponding to the spectral type and luminosity class of the considered star. Values of $T_{\text {eff }}$ and $\log \mathrm{g}$ for a given spectral type were obtained from calibration of Martins, Schaerer, \& Hillier (2005a) for O-stars and from Humphreys \& McElroy (1984) for B-stars. The chosen spectrum was convolved with the instrumental profile and rotational profile computed with different values of $v \sin i$. The convolution of spectra was performed using the ROTIN3 program (distributed together with TLUSTY).

The projected rotational velocity and its uncertainty were then estimated by visual comparison of shapes of the observed spectral line profiles of metals and helium with the ones in the convolved synthetic spectra. These estimates are independent on the microturbulent velocity $v_{\text {turb }}$ that only affects the equivalent width of line and not line profile shape.

\subsection{Determination of effective temperature and surface gravity}

To estimate $T_{\text {eff }}$ and $\log$ g, we used the automatic method. The method is based on the comparison of the observed and 
Table 1. Observational log for the spectral data obtained on the Russian 6-m telescope used in the work. The Column 1 quotes the names of the objects from the catalogue of Schulte (1956), while the Column 2-the ones from Massey \& Thompson (1991). The penultimate column lists the signal-to-noise ratio (S/N) in the vicinity of HeI $\lambda 5876$ line (for NES data) or near $4900 \AA$ (for SCORPIO).

\begin{tabular}{|c|c|c|c|c|c|c|c|c|c|}
\hline Schulte & MT & Spectral type ${ }^{a}$ & $m_{V}[\mathrm{mag}]$ & $A_{V}[\mathrm{mag}]$ & Date $\mathrm{dd} / \mathrm{mm} /$ year & Spectrograph & Spectral range, $[\AA]]$ & $\mathrm{S} / \mathrm{N}$ & Ref. $^{b}$ \\
\hline \multirow[t]{2}{*}{6} & \multirow[t]{2}{*}{317} & \multirow[t]{2}{*}{$\mathrm{O} 8 \mathrm{~V}$} & \multirow[t]{2}{*}{10.68} & \multirow[t]{2}{*}{4.6} & 03.06 .2010 & NES & $5300-6690$ & 60 & 1 \\
\hline & & & & & 04.06 .2010 & NES & $4465-5930$ & 40 & \\
\hline 16 & 299 & $\mathrm{O} 7.5 \mathrm{~V}$ & 10.84 & 4.4 & 10.06 .2011 & NES & $4850-6240$ & 60 & 1 \\
\hline \multirow[t]{3}{*}{21} & 259 & B0 V & 11.42 & 3.7 & 18.11 .2010 & NES & $5215-6690$ & 100 & 1 \\
\hline & 282 & B1 IV-V & 15.03 & 8.12 & 31.05 .2013 & SCORPIO & $4050-5850$ & 50 & 2 \\
\hline & 343 & $\mathrm{~B} 1 \mathrm{~V}$ & 14.5 & 6.76 & 03.08 .2014 & SCORPIO & $4050-5850$ & 70 & 2 \\
\hline
\end{tabular}

${ }^{a}$ Spestral types are taken from literature (see the text).

${ }^{b}$ Photometric data $m_{V}$ and $A_{V}$ are taken from 1-Kiminki et al. (2007), 2-Maryeva et al. (2016).

synthetic spectra. Synthetic spectra for different values of $T_{\text {eff }}$, $\log \mathrm{g}, v_{\text {turb }}$, and helium abundance $\mathrm{He} / \mathrm{H}$ had been computed for the models from the TLUSTY grids using SYNSPEC4 8 code (Hubeny \& Lanz 1992) and then convolved with rotational profile for the given value of $v \sin i$ and with the instrumental profile. To characterise how well the observed spectral lines or regions are approximated by model spectra, the $\chi^{2}$ quantity was evaluated as

$$
\chi^{2}=\frac{1}{N_{\text {lines }}} \sum_{i=1}^{N_{\text {lines }}} \frac{1}{N_{v}^{i}} \sum_{j=1}^{N_{v}^{i}}\left(\frac{F_{j}^{i}-F_{j \text { obs }}^{i}}{\sigma^{i}}\right)^{2},
$$

where $N_{v}^{i}$ is the number of wavelength points in the spectral line $i, N_{\text {lines }}$ is the number of spectral lines used in the analysis, $F_{j \text { obs }}^{i}$ is the observed normalised flux in the $j$-th point of the spectral line $i, F_{j}^{i}$ is the synthetic normalised flux, and $\sigma^{i}=(\mathrm{S} / \mathrm{N})^{-1}$ accounts for the $\mathrm{S} / \mathrm{N}$ for the spectral line $i$. The $\mathrm{S} / \mathrm{N}$ had been calculated as the mean flux divided by the standard deviation of the flux in the manually selected continuum region close to a given line.

Initial estimates of $T_{\text {eff }}, \log \mathrm{g}, \mathrm{He} / \mathrm{H}$, and $v_{\text {turb }}$ were obtained as parameters of the model with the minimal $\chi^{2}$ value. The synthetic spectrum computed with these initial parameters was used to manually refine the continuum (as the initial continuum estimation is being done manually and is prone to errors due to its ill-defined nature) and to check the previously estimated $v \sin i$ value. To refine the continuum, we also used the $T_{\text {eff }}-\log$ g diagnostic diagram [see e.g. Herrero et al. (1992)] plotted automatically on the basis of $\chi^{2}$ values of the models with fixed $\mathrm{He} / \mathrm{H}$ and $v_{\text {turb }}$ equal to their initial estimates.

The OSTAR2OO2 and BSTAR2O06 grids has a relatively rough step on $T_{\text {eff }}$ and $\log \mathrm{g}\left(2500 \mathrm{~K}\right.$ on $T_{\text {eff }}$ and 0.25 dex on $\left.\log \mathrm{g}\right)$ that could lead to relatively large uncertainty of estimated $T_{\text {eff }}$ and $\log g$ values. To refine $T_{\text {eff }}$ and $\log g$ estimates obtained at the previous step, we computed the grid of stellar atmosphere models with varied values of $T_{\text {eff }}$ and $\log g$ and with fixed $\mathrm{He} / \mathrm{H}$ and $v_{\text {turb }}$ values. These fixed values were close to those obtained on the previous step. The new grid had steps of $1000 \mathrm{~K}$ and 0.1 dex on $T_{\text {eff }}$ and $\log$ g, respectively. Computations of the new grid were performed with the TLUSTY2OO code that was slightly modified to allow using of Kurucz (1994) line data for nickel (Ni). Atomic models were the same as for stellar atmosphere models from the OSTAR2OO2 grid. To save computer time, all stellar atmosphere models for the new grid were computed with the smaller number of lines of iron-peak elements, with smaller wavelength range and smaller maximum number of global iterations compared with those used by Lanz \& Hubeny (2003).

The new atmosphere models grid was used to compute a number of synthetic spectra for different values of $T_{\text {eff }}$, $\log \mathrm{g}, \mathrm{He} / \mathrm{H}$, and $v_{\text {turb }}$. These synthetic spectra were compared with observed ones using the $\chi^{2}$ quantity. To obtain resulting estimates of the varied stellar parameters, we have selected all models with $\chi^{2}$ smaller than the threshold value $\chi_{\mathrm{t}}^{2}$ :

$$
\chi_{\mathrm{t}}^{2}=\chi_{\min }^{2}+0.3 \chi_{\min }^{2}
$$

where $\chi_{\min }^{2}$ is the minimal $\chi^{2}$ value. A very similar criterion for the selection of models, with a bit different threshold value, was used by Castro et al. (2012) for the analysis of their low resolution spectra. Our choice of $\chi_{t}^{2}$ threshold is discussed in the Appendix A. Resulting values of $T_{\text {eff }}, \log \mathrm{g}$, $\mathrm{He} / \mathrm{H}$, and $v_{\text {turb }}$ were obtained by averaging parameters of selected models weighted by $\exp \left(-0.5 \chi^{2}\right)$. Application of such weights implies normal distribution of $\chi^{2}$ that is the good assumption when the number of degrees of freedom is large. Errors of stellar parameters were obtained as weighted standard deviations of parameters of selected models. The spectrum computed with parameters closest to resulting ones was then used again to check the continuum and to verify $v \sin i$ value. If needed, we repeated the estimation of parameters with the stellar atmospheres grid computed earlier.

The method of automatic determination of $T_{\text {eff }}$ and $\log g$ was common for both high and low resolution observed spectra. In case of low resolution model spectra were convolved with instrumental profile only, neglecting $v \sin i$, since instrumental broadening dominates over rotational broadening. Moreover, stellar atmosphere models with smaller steps on $T_{\text {eff }}$ and $\log g$ were not computed. The testing of the method for both high- and low-resolution spectra is presented in the Appendix B. 


\subsection{Determination of bolometric luminosity and mass-loss rate}

As mentioned above, for determination of wind parameters and bolometric luminosity, we used models computed with CMFGEN code (Hillier \& Miller 1998). Every model is defined by a hydrostatic stellar radius $R_{*}$, luminosity $L_{*}$, mass-loss rate $\dot{M}$, filling factor $f$, wind terminal velocity $V_{\infty}$, stellar mass $M_{*}$, and by the abundances $Z_{i}$ of contained elementary species. We included $\mathrm{H}, \mathrm{He}, \mathrm{C}, \mathrm{N}, \mathrm{O}, \mathrm{Si}, \mathrm{S}, \mathrm{P}, \mathrm{Mg}, \mathrm{Al}$, and $\mathrm{Fe}$ in calculations.

For the photospheric density structure, we used the hydrostatic density structure computed with WIND_HYD program (one of CMFGEN utilities). For the wind, we used a standard $\beta$-velocity law $\left(V=V_{\infty}\left(1-\frac{R_{*}}{r}\right)^{\beta}\right)$ that is connected to the hydrostatic density structure just above the sonic point. We chose $\beta=1.0$ as the default value for our calculation since it turns out to be typical for O-dwarfs [e.g. Massa et al. (2003); Martins et al. (2005b)]. We did not incorporate the clumping into our models, as the spectra we use lack wind emission lines.

The first important step is the determination of a bolometric luminosity. Due to the fact that the stars belong to Cyg OB2 association, distance for which is known, we were able to estimate their luminosities. Distance to Cyg OB2 was estimated in various works using different methods. For example, spectrophotometric distance measurements span a range that includes $1.5 \mathrm{kpc}$ (Johnson \& Morgan 1954), $2.1 \mathrm{kpc}$ (Reddish, Lawrence, \& Pratt 1966), $1.7 \mathrm{kpc}$ (Massey \& Thompson 1991; Torres-Dodgen, Carroll, \& Tapia 1991), and $1.45 \mathrm{kpc}$ (Hanson 2003). Using double-lined eclipsing binaries, Kiminki et al. (2015) found that the distance is $1.33 \mathrm{kpc}$. In this work as well as in previous studies (Maryeva et al. 2013, 2014), we used an estimation of distance $d=$ $1.5 \mathrm{kpc}$ from Mel'nik \& Dambis (2009), who acquired it from the analysis of line-of-sight velocities and proper motions of OB-associations. Note that the uncertainty of distance of $0.1 \mathrm{kpc}$ results in uncertainty of model stellar magnitude of about $0.14 \mathrm{mag}$, which is comparable to the errors of apparent stellar magnitudes.

For each sample star, we took model from 'A grid of O star CMFGEN Models' ${ }^{3}$ closest to the star in $T_{\text {eff }}$ and $\log \mathrm{g}$. We changed bolometric luminosities and iteratively refined the model in a such way that model photometry matched the observational data $\left(m_{V}\right.$ from Table 1$)$. Model photometry means magnitude in $V$ filter derived from model spectrum, recalculated for the distance to the Cyg OB2 association and corrected for the interstellar extinction. The values of the interstellar extinction were taken from Kiminki et al. (2007) and Maryeva et al. (2016), they are listed in Table 1. On the next step, the resulted model was used as the seed model. Then, we adjusted the parameters of model including temperature by means of qualitative comparison of computed spectra with observations.

\footnotetext{
${ }^{3}$ http://kookaburra.phyast.pitt.edu/hillier/web/CMFGEN.htm
}

For measuring $T_{\text {eff }}$ with CMFGEN, we compared intensities of different ion (CII,III,IV; NII,III, HeI,II) lines, i.e. we used the traditional ionisation-balance method. CMFGEN measurements of $T_{\text {eff }}$ are in a good agreement with TLUSTY estimations.

For all sample stars, there are no estimations of $V_{\infty}$. Therefore for each stars, we set the value of $V_{\infty}=2.65 V_{\text {esc }}(\mathrm{Ku}-$ dritzki \& Puls 2000) and measured an upper limit of massloss rate by visual comparison of model spectra with observational data.

\section{RESULTS}

Projected rotational velocities $v \sin i$ of studied stars have not been estimated previously. The exception is MT259 for which Herrero et al. (1999) estimated $v \sin i=30 \mathrm{~km} \mathrm{~s}^{-1}$. High resolution spectra of MT259, MT299, and MT317 allowed us to measure the $v \sin i$ of these stars. Table 2 gives measured values of $v \sin i$ which were used for determination of other parameters and shows that our estimate is well consistent with one by Herrero et al. (1999).

We determined $T_{\text {eff }}, \log \mathrm{g}, L_{*}$, and other parameters of the sample stars using the methods described in Sections 3.2 and 3.3. Spectral lines used to estimate $T_{\text {eff }}$ and $\log g$ of MT259, MT299, and MT317 with TLUSTY are listed in Table 3. On the other hand, for the determination of parameters with CMFGEN we compared the overall shape of the model spectra with observational one, not just the separate lines. Figures 1-3 show the comparison of several lines from the spectra of MT259, MT299, and MT317 with model ones. They also demonstrate that both CMFGEN and TLUSTY successfully describe observed lines. We do not list metal lines used to estimate $v \sin i$ in Table 3 but show some of these lines in the figures.

Due to the low-spectral resolution of our spectra for MT282 and MT343, we were unable to estimate their $v \sin i$. Moreover, in our spectra of MT282 and MT343 there are no lines of ionised helium, and the silicon lines are not well resolved. Thus, it is problematic to constrain the effective temperature simultaneously along with other parameters using the automatic method. To avoid such problems, we varied only $T_{\text {eff }}$ and $\log$ g. We also considered not only spectral lines but spectral regions which contains lines useful to constrain $T_{\text {eff }}$. In this study, we selected the region $\lambda \lambda 4547-4591$ containing SiIII lines and the region $\lambda \lambda 4665-4731$ around HeII $\lambda 4686$ line (see Table 3 ). The values of $T_{\text {eff }}$ and $\log g$ determined with TLUSTY became starting parameters for CMFGEN calculations, results of which are presented in Figure 4. For calculation of their $V_{\infty}$, we supposed that $\log \mathrm{g}=3.9$ and $M_{*}$ are $20.6 \mathrm{M}_{\odot}$ and $13 \mathrm{M}_{\odot}$ for MT282 and MT343 accordingly.

The derived parameters are presented in Table 2. For comparison, Table 2 also gives the parameters of MT259 estimated by Herrero et al. (1999). The value of $T_{\text {eff }}$ obtained in this study $(31.2 \mathrm{kK})$ is lower than the one given by Herrero et al. (1999) (34.5 kK), and we will discuss it below. The 
Table 2. Physical parameters and wind properties of the studied Cyg OB2 stars. $R_{2 / 3}$ is the radius where the Rosseland optical depth is equal to $2 / 3, T_{\text {eff }}$ is the effective temperature at $R_{2 / 3}$. The last column gives values of upper limits for mass-loss rate.

\begin{tabular}{|c|c|c|c|c|c|c|c|c|c|c|}
\hline Star & $\begin{array}{l}\text { Spec. } \\
\text { class }^{a}\end{array}$ & $\log \mathrm{L} * / \mathrm{L}_{\odot}$ & $T_{\text {eff }}[\mathrm{kK}]$ & $R_{2 / 3}\left[\mathrm{R}_{\odot}\right]$ & $M_{*}\left[\mathrm{M}_{\odot}\right]$ & $\log g$ & $\begin{array}{c}v \sin i \\
{\left[\mathrm{~km} \mathrm{~s}^{-1}\right]}\end{array}$ & $v_{\text {turb }}\left[\mathrm{km} \mathrm{s}^{-1}\right]$ & $V_{\infty}\left[\mathrm{km} \mathrm{s}^{-1}\right]$ & $\begin{array}{l}\dot{M} \cdot 10^{-7} \\
{\left[\mathrm{M}_{\odot} \mathrm{yr}^{-1}\right]}\end{array}$ \\
\hline MT259 & B $0 \mathrm{~V}$ & $4.4 \pm 0.04$ & $31.2 \pm 0.7$ & 5.3 & $10.3 \pm 4$ & $3.97 \pm 0.16$ & $25 \pm 5$ & $9 \pm 1$ & 2230 & 0.3 \\
\hline MT282 & B1 IV & $4.4 \pm 0.04$ & $25 \pm 3$ & 8.42 & $<26$ & $3.9 \pm 0.3$ & & & 2560 & \\
\hline MT299 & $07.5 \mathrm{~V}$ & $4.93 \pm 0.04$ & $33.3 \pm 1.1$ & 8.75 & $17.7 \pm 7$ & $3.78 \pm 0.21$ & $200 \pm 20$ & $12 \pm 3$ & 2280 & 1.5 \\
\hline МТ317 & O8 IV & $5.08 \pm 0.04$ & $32.8 \pm 1.0$ & 10.6 & $23 \pm 10$ & $3.72 \pm 0.21$ & $210 \pm 20$ & $12 \pm 3$ & 2370 & 3.5 \\
\hline MT343 & B1 V & $4.3 \pm 0.04$ & $26 \pm 3$ & 6.7 & $<16.4$ & $3.9 \pm 0.3$ & & & 2280 & \\
\hline $\mathrm{MT} 259^{b}$ & & & 34.5 & & & 3.9 & 30 & & & \\
\hline
\end{tabular}

${ }^{a}$ Spectral types according to our estimations of luminosities.

${ }^{b}$ Data are taken from Herrero et al. (1999).
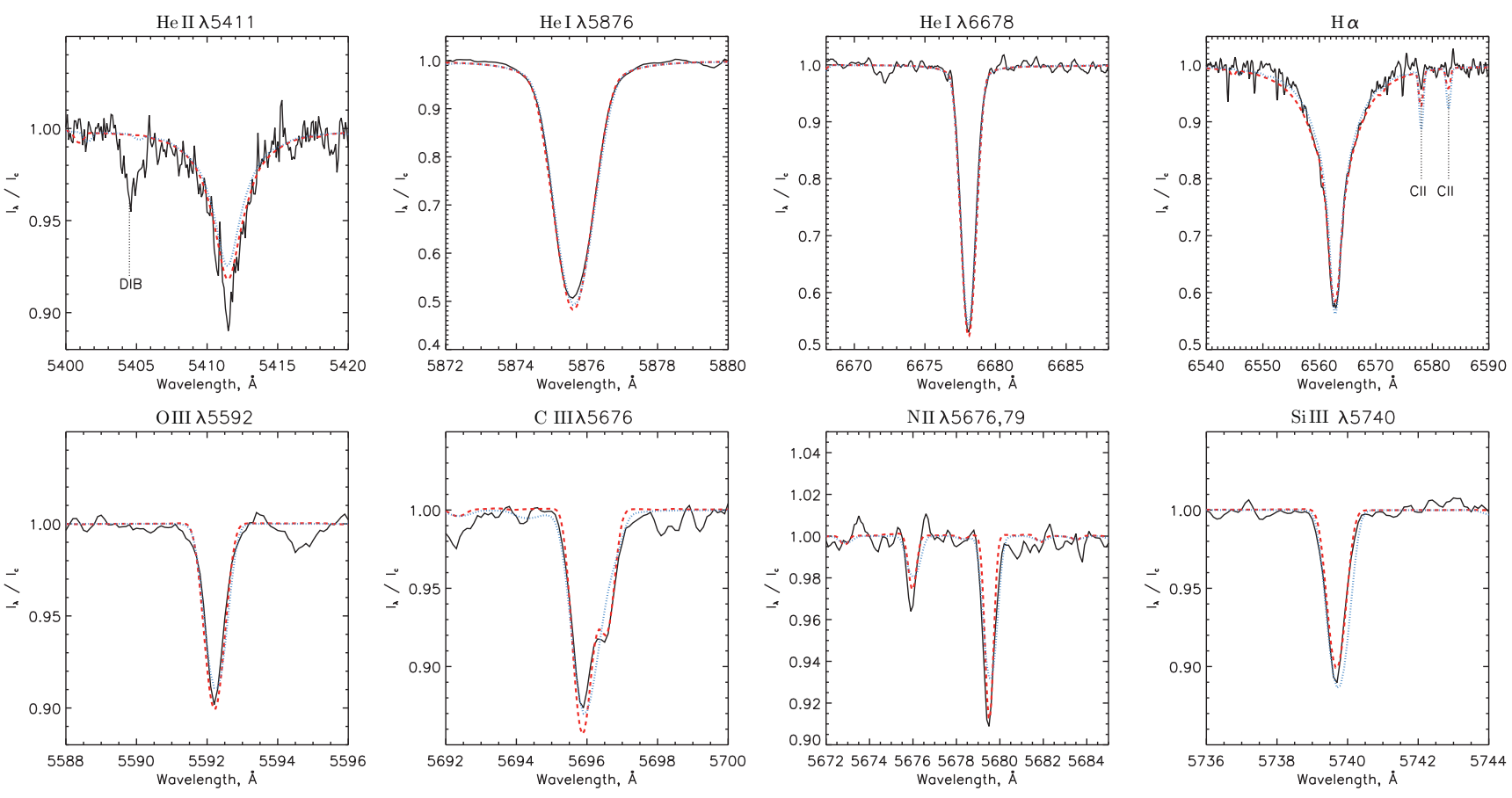

Figure 1. Modelling of MT259 (B0 V). The solid line shows the observed profile, the blue-dotted line-the TLUSTY-model and the red-dashed line - the CMFGEN-model.

other parameters of MT259 do agree with results of Herrero et al. (1999).

All optical helium lines falling into the observed spectral range were used to constrain the relative $\mathrm{He}$ to $\mathrm{H}$ abundance. As a result, all Cyg OB2 stars studied in this work demonstrate nearly solar helium abundance ${ }^{4} \mathrm{He} \simeq 26 \%$ $(\mathrm{He} / \mathrm{H}=0.09 \pm 0.01)$.

We determined the $\mathrm{C}, \mathrm{N}$, and $\mathrm{O}$ abundances for MT259 and MT317 using CII $\lambda \lambda 6578,6583$, CIII $\lambda 5826$, CIV $\lambda \lambda 5801.3,5812, \quad$ NII $\quad \lambda \lambda 5666,5676,5680,5686$, OIII $\lambda 5592.25$ for the first star, and CIV $\lambda \lambda 5801.3,5812$, NIII $\lambda \lambda 4511,4515,4634.0,4640.6$, OIII $\lambda 5592.25$ for the second. Calculated abundances of the elements are given in Table 4. Figure 5 shows the locations of MT259 and MT317 on $\log (N / C) / \log (N / O)$ diagram, taken from Martins et al.

${ }^{4}$ Solar He abundance is $\mathrm{He} / \mathrm{H}=0.085 \pm 0.002$ according to Grevesse, Asplund, \& Sauval (2007).
Table 3. Spectral lines and regions used to estimate $T_{\text {eff }}$ and $\log g$ of considered Cyg OB2 stars with TLUSTY atmosphere models.

\begin{tabular}{lc}
\hline \hline Star & Spectral lines and regions used in the analysis \\
\hline MT259 & HeI $\lambda \lambda 5876,6678$ HeII $\lambda 5411$ wings of $\mathrm{H} \alpha$ \\
MT299 & HeI $\lambda \lambda 4921,5015,5876$, HeII $\lambda 5411$ wings of $\mathrm{H} \beta$ \\
MT317 & HeI $\lambda \lambda 4713,4921,5015,5876$ \\
& HeII $\lambda \lambda 4542,4686,5411$ wings of $\mathrm{H} \alpha$ and $\mathrm{H} \beta$ \\
MT282 & $\mathrm{HeI} \lambda \lambda 4143,4388,4471,4921,5015$ \\
& $\mathrm{H} \beta, \mathrm{H} \gamma, \mathrm{H} \delta, \mathrm{CIII} \lambda 4650$ \\
& $\lambda \lambda 4547-4591, \lambda \lambda 4665-4697$ \\
MT343 & $\mathrm{HeI} \lambda \lambda 4143,4388,4471,4921,5015$ \\
& $\mathrm{H} \beta, \mathrm{H} \gamma, \mathrm{H} \delta, \mathrm{CII} \lambda 4267, \mathrm{CIII} \lambda 4650$ \\
& $\lambda \lambda 4547-4592, \lambda \lambda 4665-4731$ \\
\hline \hline
\end{tabular}

(2015) (the figure 10 in the original paper). Although MT259 and MT317 are members of the association and lie farther from us than stars considered in the study of Martins 

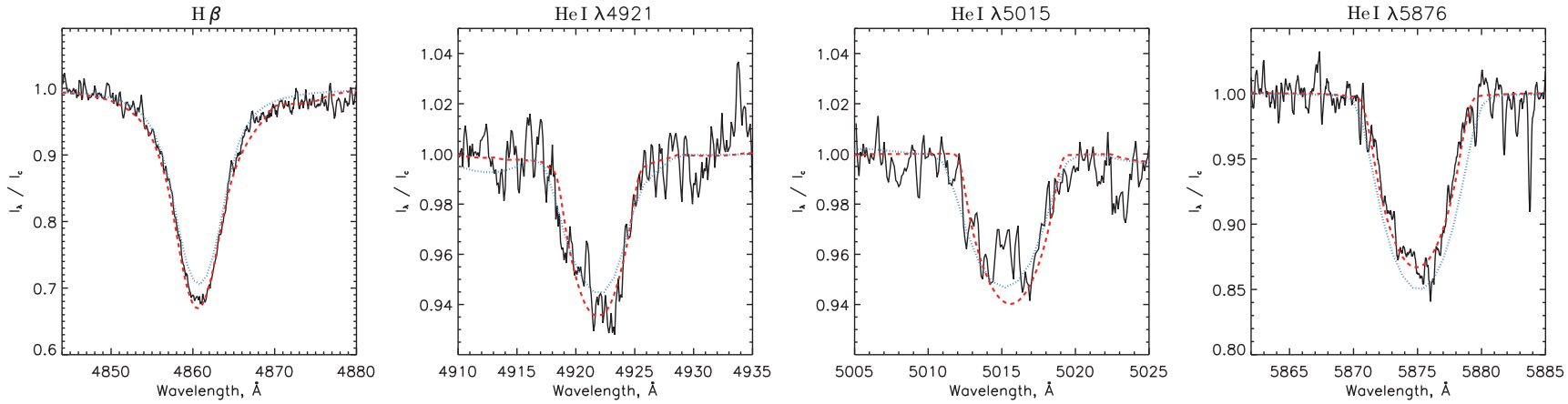

Figure 2. Modelling of MT299 (O7.5 V). The solid line shows the observed profile, the blue-dotted line—-the TLUSTY-model and the red-dashed line-the CMFGEN-model.
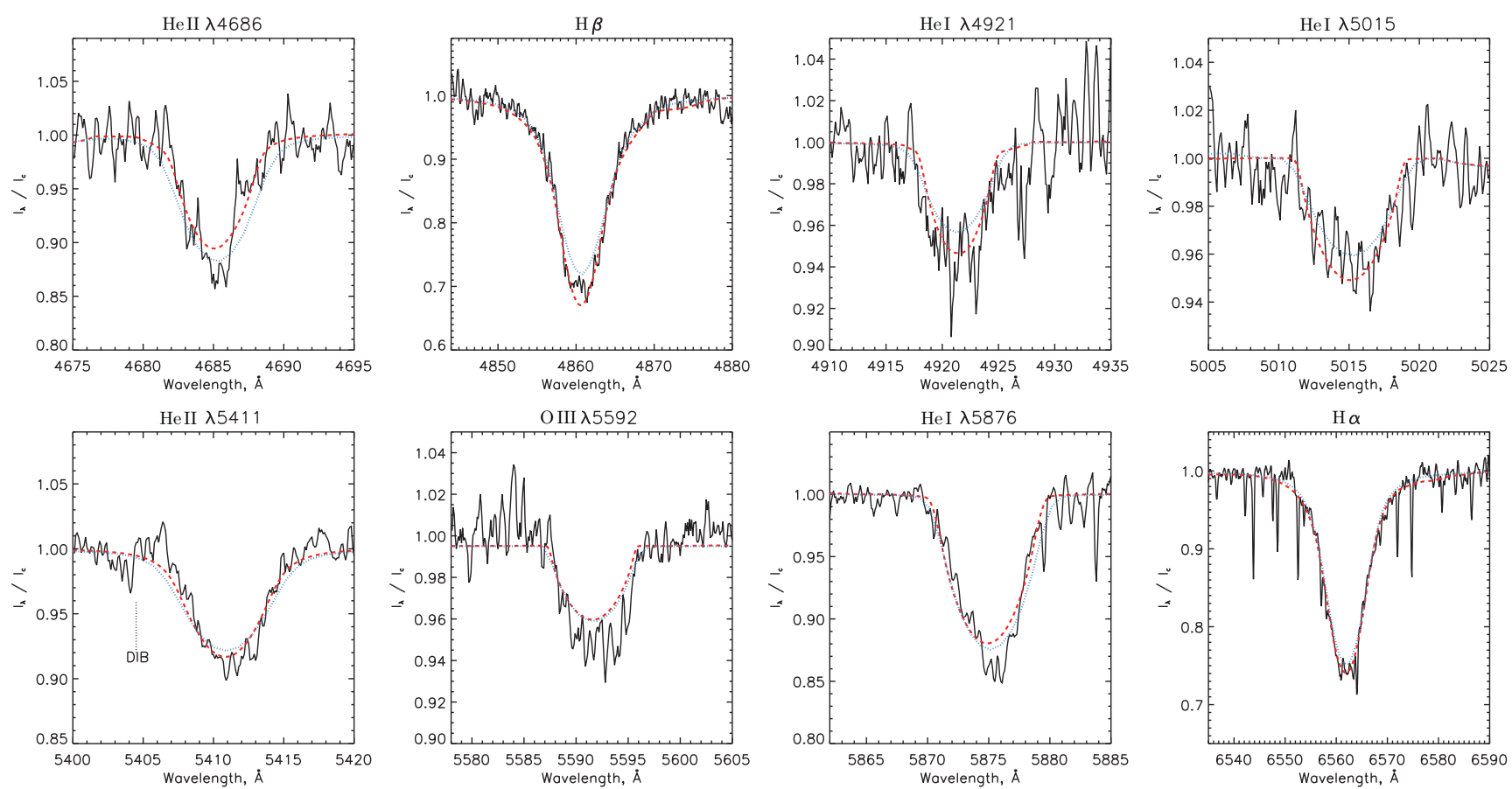

Figure 3. Modelling of MT317 (O8 IV). Comparison of the profiles of selected lines with the best model spectra. The solid line shows the observed profile, the blue-dotted line-the TLUSTY-model and the red-dashed line-the CMFGEN-model.

Table 4. The abundances of chemical elements estimated with CMFGEN atmosphere models.

\begin{tabular}{lcccc}
\hline \hline Star & $\mathrm{He} / \mathrm{H}$ & $\mathrm{C} / \mathrm{H}\left[10^{-4}\right]$ & $\mathrm{N} / \mathrm{H}\left[10^{-4}\right]$ & $\mathrm{O} / \mathrm{H}\left[10^{-4}\right]$ \\
\hline MT259 & $0.09 \pm 0.01$ & $2 \pm 1$ & $0.8 \pm 0.1$ & $3.8 \pm 1$ \\
MT317 & $0.09 \pm 0.01$ & $5 \pm 2$ & $2 \pm 1$ & $7 \pm 2$ \\
\hline \hline
\end{tabular}

et al. (2015), they do not show any anomalies of chemical composition.

For MT282, MT343, and MT299, however, we were not able to derive the chemical composition from our spectra due to limited spectral range of our data not including all the necessary diagnostic lines together. Due to that, for the estimation of physical parameters we assumed that chemical composition of MT282 and MT343 is the same as of MT259, while C, N, and O abundances in MT299 is equal to one of MT317.

\section{DISCUSSION}

\subsection{Temperatures and luminosities}

As it was mentioned in Section 4, the value of $T_{\text {eff }}$ obtained in this study for MT259 is lower than the one given by Herrero et al. (1999), although it is close to estimate of Humphreys \& McElroy (1984) and calibration of Crowther (1997) for B0 V spectral type. This may be related to the difference of stellar atmosphere models used by us and used by Herrero et al. (1999)—our models, for example, do account for line 

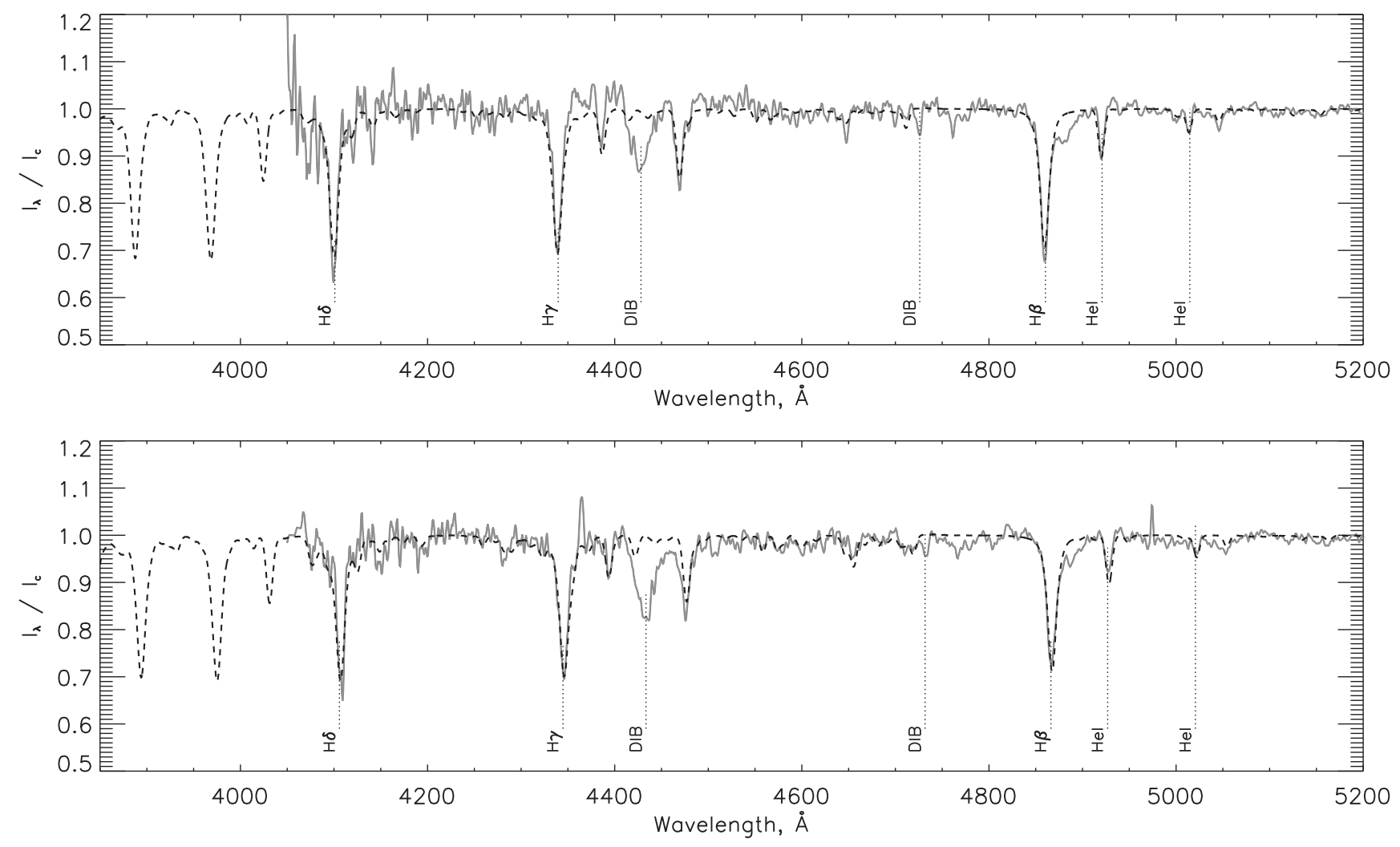

Figure 4. Comparison of the observed continuum normalised spectra of MT282 (top) and MT343 (bottom) with the CMFGEN-model (dashed line).

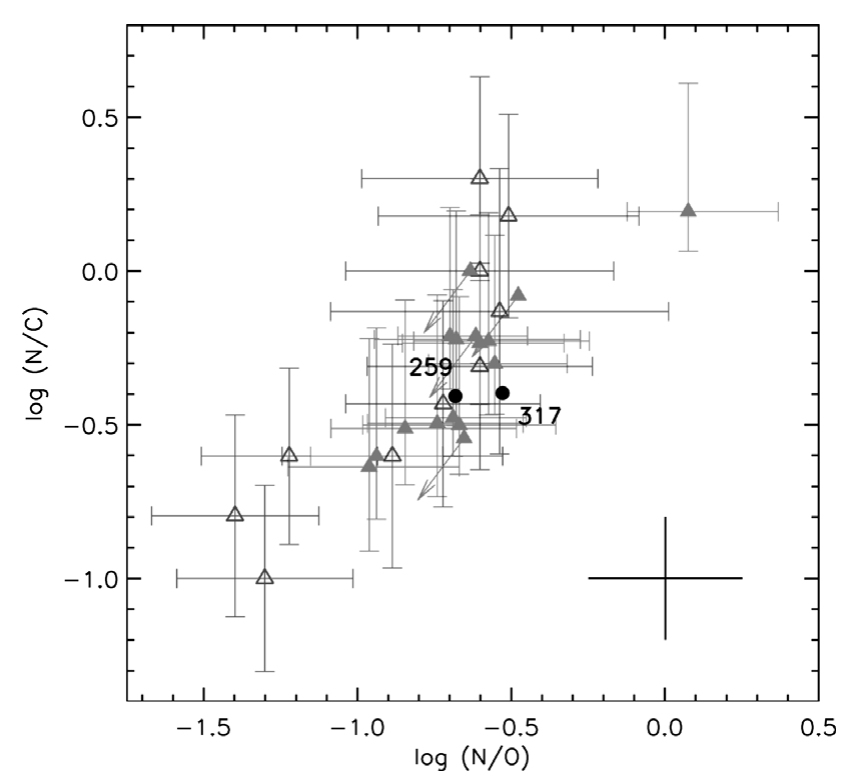

Figure 5. $\log (N / C)$ as a function of $\log (N / O)$ for dwarfs stars. Circles are the studied stars; filled triangles are Galactic stars from Martins et al. (2015); open triangles are SMC dwarfs from Bouret et al. (2013). The black cross in right lower corner displays typical errors of estimated abundances.

blanketing. As it was shown by Repolust, Puls, \& Herrero (2004), $T_{\text {eff }}$ values obtained with line-blanketed models are lower than values obtained with unblanketed stellar atmosphere models.
Martins et al. (2005a) presented new calibrations of stellar parameters of O-stars as a function of spectral type based on atmosphere models computed with the CMFGEN code. They derived two types of effective temperature scales: an $o b$ servational one from the results of modelling of individual O-stars and a theoretical one based on the grid of models. We compared our O-stars MT299 (O7.5 V) and MT317 (O8 IV) with these scales. Values of $T_{\text {eff }}$ estimated by us for MT299 and MT317 are slightly lower than the values that could be obtained from spectral classification of these stars and observational $T_{\text {eff }}$ scale of Martins et al. (2005a). If we use theoretical $T_{\text {eff }}$ scale of Martins et al. (2005a) then our $T_{\text {eff }}$ estimates are in better agreement with spectral class.

The modelling we performed allows us to refine the luminosity classes of studied stars. There is uncertainty in luminosity class of MT259. Kiminki et al. (2007) estimated that MT259 is a supergiant (B0 Ib) while Chentsov et al. (2013) claims that this star is a dwarf (B0 V). Our estimates of log $\mathrm{g}$ and $L_{*}$ for this star confirm the classification of Chentsov et al. (2013). Luminosity of MT317 is a bit higher than the tabulated value of luminosity for $\mathrm{O} 8 \mathrm{~V}$ class, according to Martins et al. (2005a). Probably MT317 should be classified as O8 IV. Maryeva et al. (2016) claimed that the absorption lines in the spectrum of MT282 are not so deep as ones in the spectrum of MT343 (classified as B1 V dwarf), and probably the stars differ in luminosity class. Our modelling also demonstrated that MT282 is brighter than MT343, and 
Table 5. Derived masses, luminosities, and ages of stars considered in this study, $*$-spectroscopic, evol-evolutionary. Spectral classes are according to our estimations of luminosities. $\dot{M}$ are upper limits for mass-loss rate.

\begin{tabular}{|c|c|c|c|c|c|}
\hline $\begin{array}{l}\text { Star } \\
\text { Spec. class }\end{array}$ & $\begin{array}{l}\text { MT259 } \\
\text { B0 V }\end{array}$ & $\begin{array}{l}\text { MT282 } \\
\text { B1 IV }\end{array}$ & $\begin{array}{l}\text { MT299 } \\
\text { O7.5 V }\end{array}$ & $\begin{array}{l}\text { MT317 } \\
\text { O8 IV }\end{array}$ & $\begin{array}{l}\text { MT343 } \\
\text { B1 V }\end{array}$ \\
\hline \multicolumn{6}{|c|}{ Measured using CMFGEN } \\
\hline $\log L * / \mathrm{L}_{\odot}$ & $4.4 \pm 0.04$ & $4.4 \pm 0.04$ & $4.93 \pm 0.04$ & $5.08 \pm 0.04$ & $4.3 \pm 0.04$ \\
\hline $\log T_{\text {eff }}$ & $4.5 \pm 0.01$ & $4.4 \pm 0.05$ & $4.522 \pm 0.013$ & $4.516 \pm 0.013$ & $4.42 \pm 0.05$ \\
\hline$M_{V}$ & -3.27 & -3.8 & -4.49 & -4.89 & -3.42 \\
\hline$B C$ & -2.9 & -2.37 & -3 & -2.98 & -2.5 \\
\hline$M_{*},\left[\mathrm{M}_{\odot}\right]$ & $10 \pm 4$ & $<26$ & $18 \pm 7$ & $23 \pm 10$ & $<16.4$ \\
\hline$\dot{M},\left[\mathrm{M}_{\odot} \mathrm{yr}^{-1}\right]$ & $3 \cdot 10^{-8}$ & & $1.5 \cdot 10^{-7}$ & $3.5 \cdot 10^{-7}$ & \\
\hline$\dot{M}_{\mathrm{Vink}},\left[\mathrm{M}_{\odot} \mathrm{yr}^{-1}\right]$ & $8.4 \cdot 10^{-9}$ & & $1.44 \cdot 10^{-7}$ & $2.1 \cdot 10^{-7}$ & \\
\hline \multicolumn{6}{|c|}{ Measured using $\left(\log g, T_{\text {eff }}\right)$} \\
\hline$M_{\mathrm{evol}},\left[\mathrm{M}_{\odot}\right]$ & $18_{-1}^{+2}$ & $11 \pm 1$ & $24_{-2}^{+4}$ & $24_{-2}^{+4}$ & $12 \pm 1$ \\
\hline $\log L_{\text {evol }} / L_{\odot}$ & $4.63 \pm 0.05$ & $4.05 \pm 0.05$ & $5.03 \pm 0.07$ & $5.06 \pm 0.07$ & $4.18 \pm 0.05$ \\
\hline Age, $[\mathrm{Myr}]$ & $5.9 \pm 0.9$ & $14 \pm 2$ & $5.6 \pm 0.4$ & $5.9 \pm 0.4$ & $12.1 \pm 1.5$ \\
\hline \multicolumn{6}{|c|}{ Measured using $\left(L_{*}, T_{\text {eff }}\right)$} \\
\hline$M_{\mathrm{evol}},\left[\mathrm{M}_{\odot}\right]$ & $16 \pm 1$ & $12.9 \pm 0.5$ & $22 \pm 1$ & $24 \pm 1$ & $12.7 \pm 0.5$ \\
\hline Age,[Myr] & $1-4.5$ & $14.1 \pm 1.3$ & $5.1 \pm 0.7$ & $5.8 \pm 0.4$ & $12 \pm 2$ \\
\hline \multicolumn{6}{|c|}{ Measured by Wright et al. (2015) using M-K classification } \\
\hline $\log L_{\text {evol }} / \mathrm{L}_{\odot}$ & $4.2_{-0.17}^{+0.14}$ & & $4.92_{-0.06}^{+0.05}$ & $5.04_{-0.07}^{+0.04}$ & $4_{-0.2}^{+0.13}$ \\
\hline $\log T_{\text {eff evol }}$ & $4.44_{-0.08}^{+0.06}$ & & $4.54 \pm 0.02$ & $4.54 \pm 0.02$ & $4.39_{-0.06}^{+0.2}+08$ \\
\hline$M_{\mathrm{evol}},\left[\mathrm{M}_{\odot}\right]$ & $12.9_{-3.1}^{+1.3}$ & & $23.4_{-3.2}^{+1.1}$ & $24.8_{-3.4}^{+1.7}$ & $10.6_{-2.1}^{+1.2}$ \\
\hline Age, $[\mathrm{Myr}]$ & $7.63_{-4.46}^{+2.37}$ & & $3.65_{-2.35}^{+1.07}$ & $4.28_{-2.4}^{+0.75}$ & $>10$ \\
\hline
\end{tabular}

argued in favour of MT282 being the star of B1 IV type. Tables 2 and 5 give the refined luminosity classes.

\subsection{Ages and masses}

Estimations of masses and ages are important for reconstruction of star-formation history in the Cyg OB2 region, they are also necessary to define the initial mass function (IMF). Accurate determination of mass and age requires knowing precise location of the star on $\mathrm{H}-\mathrm{R}$ diagram, which is often being done based on the tabulated values for a given spectral class in Morgan-Keenan (M-K) system. This leads to uncertainties due to inevitable difference between tabulated data and intrinsic luminosities and temperatures of stars. According to Wright et al. (2015), for O-type stars these uncertainties are typically small, $\sim 0.02 \mathrm{dex}$ in $\log \mathrm{T}_{\text {eff }}$ and $\sim 0.1 \mathrm{dex}$ in $\log \mathrm{L} * / \mathrm{L}_{\odot}$. However for the $\mathrm{B}$-type stars, the errors rise significantly to $\sim 0.07$ dex in $\log \mathrm{T}_{\text {eff }}$ and $\sim 0.2 \mathrm{dex}$ in $\log \mathrm{L} * / \mathrm{L}_{\odot}$ due to classification uncertainties and the larger difference in $T_{\text {eff }}$ between spectral subclasses. Our uncertainties obtained as result of modelling are significantly smaller: $<0.014 \mathrm{dex}$ in $\log \mathrm{T}_{\text {eff }}$ and $<0.04$ dex in $\log \mathrm{L} * / \mathrm{L}_{\odot}$ for O-type stars; $<0.02 \mathrm{dex}$ in $\log \mathrm{T}_{\text {eff }}$ and $<0.04 \operatorname{dex}$ in $\log \mathrm{L} * / \mathrm{L}_{\odot}$ for the B-type stars. Table 5 clearly demonstrates that the uncertainties in $\log \mathrm{L} * / \mathrm{L}_{\odot}$ and $\log \mathrm{T}_{\text {eff }}$ obtained with modelling are smaller than the ones obtained with $\mathrm{M}-\mathrm{K}$ classification (Wright et al. 2015).

Figures 6 and 7 show the location of studied stars in the $\mathrm{H}-$ $\mathrm{R}$ diagram and $\log g-T_{\text {eff }}$ diagram as well as evolutionary tracks and isochrones from the Geneva database (Ekström

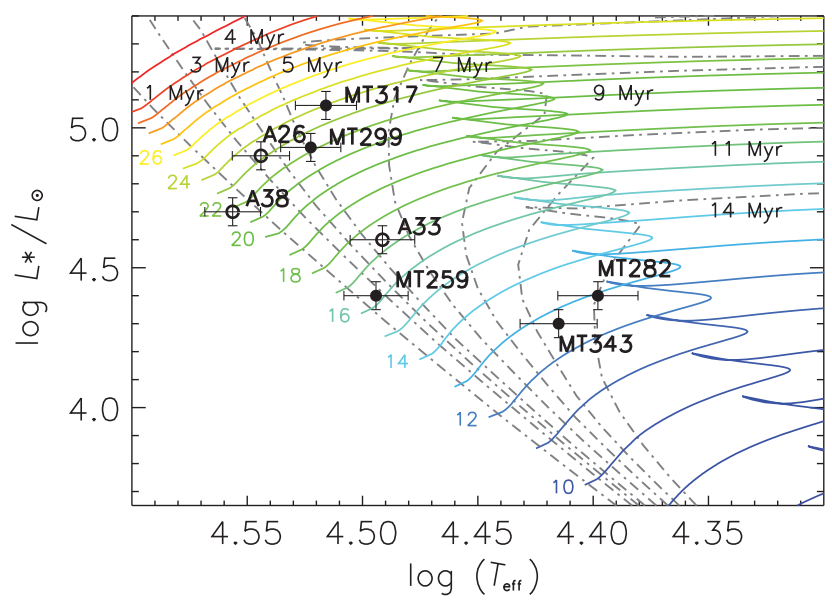

Figure 6. H-R diagram for the stars of the present study (filled circles) and the OB-dwarfs A26 (O9.5 V), A33 (B0.2 V), and A38 (O8 V) from Negueruela et al. (2008) (open circles). Evolutionary tracks (solid lines) and isochrones (dashed-dotted lines) are from Ekström et al. (2012).

et al. 2012), constructed using the online calculator ${ }^{5}$ taking into account the effects of rotation. The rotation rate is $V_{\text {ini }} / V_{\text {crit }}=0.4$.

Table 5 gives the comparison of the values of mass, luminosity, and age for each star, estimated using the diagrams and from the spectral modelling. The index 'evol' means that the quantity is measured using the $\mathrm{H}-\mathrm{R}$ or $\log g-T_{\text {eff }}$ diagram and evolutionary tracks. Also Table 5 gives the same

\footnotetext{
${ }^{5}$ http://obswww.unige.ch/Recherche/evol/-Database-
} 


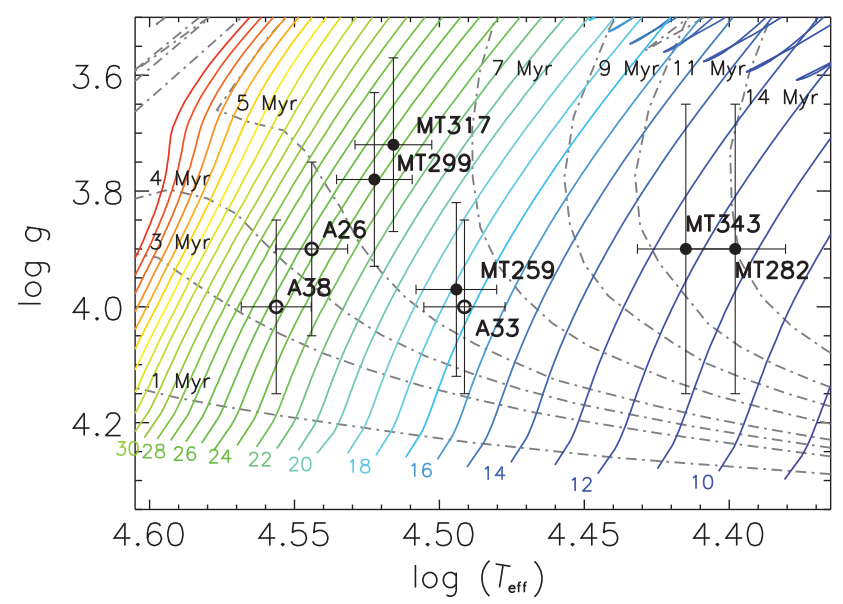

Figure 7. $\log g, T_{\text {eff }}$ diagram for the stars of the present study (filled circles) and the OB-dwarfs A26 (O9.5 V), A33 (B0.2 V) and A38 (O8 V) from Negueruela et al. (2008) (open circles). Evolutionary tracks (solid lines) and isochrones (dashed-dotted lines) are from Ekström et al. (2012).

parameters obtained from the $\mathrm{H}-\mathrm{R}$ diagram according to $\mathrm{M}-$ K classification (Wright et al. 2015). As we can see from the table, the $\mathrm{H}-\mathrm{R}$ and $\log g-T_{\text {eff }}$ diagrams provide similar values of masses and close values of ages. The luminosities estimated with CMFGEN $L_{*}$ and with $\log g-T_{\text {eff }}$ diagram $L_{\text {evol }}$ are relatively close. It once again shows that $\log g-T_{\text {eff }}$ together with the current models of stellar evolution is very useful instrument for determination of masses and ages of stars with unknown distances. The $\log g-T_{\text {eff }}$ diagram combined with isochrones provides ages, and together with evolution tracks-gives stellar mass, and therefore stellar luminosity $L_{\text {evol }}$.

From the Table 5, we also can see that $M_{*}$ is equal to $M_{\text {evol }}$ within the errors. It is also seen that $M_{*}$ tends to be lower than $M_{\mathrm{evol}}$. This tendency can be due to the so-called mass discrepancy — systematic overestimate of evolutionary masses $M_{\text {evol }}$ compared to spectroscopically derived masses $M_{*}$ [see e.g. Herrero et al. (1992)]. Markova \& Puls (2015) suggested that the reason for this effect is the neglection of turbulent pressure in FASTWIND and CMFGEN atmospheric models. Moreover, in our case the tendency is less pronounced for the stars with higher masses. This can be consistent with trends founded by Markova \& Puls (2015). But it is hard to make any certain conclusions taking into account that the uncertainties in $M_{*}$ are significantly higher than ones in $M_{\text {evol }}$. The uncertainties in $M_{*}$ result from uncertainties in $\log g$ (about $0.2 \mathrm{dex}$ ), which are linked with narrow spectra range and, therefore, small number of spectral lines, in particular, hydrogen lines used in our analysis. The increase of analysed spectral range is the way to improve the accuracy.

Massey \& Thompson (1991); Hanson (2003) have noted that the star formation in Cyg OB2 is non-coeval. Drew et al. (2008) found clustering of A-type stars at distance of 20 arcmin south of the centre of Cyg OB2 using data from the INT/WFC Photometric H $\alpha$ Survey (IPHAS). One of the interpretations is that the cluster of A-type stars and the already known OB-star concentration are parts of the same association. And probably there were two main episodes of star formation, or there is a substantial age spread (Drew et al. 2008). Wright et al. (2010) analysed the properties of stars in two fields in Cyg OB2 using deep Chandra Xray point sources catalogue and found that these two fields are different by age of star generation. Comerón \& Pasquali (2012) concluded that members of the association display a mixture of ages ranging from less than $3 \mathrm{Myr}$ to over $10 \mathrm{Myr}$, indicating a long sustained rate of star formation. Also Comerón \& Pasquali (2012) claimed that Cyg OB2 extends beyond the area occupied by the youngest and hottest stars and that the southern part of the association is clearly older. Wright et al. (2015) presented list of massive stars in Cyg OB2 which is most complete as of now. The age distribution of stars more massive than $20 \odot$ and down to $\sim$ B0.5 V spectral type showed that star formation process started at least $\sim 6-8 \mathrm{Myr}$ ago. Between 1 and 7 Myr ago, star formation occurred more or less continuously with probable excess between 4-5 Myr ago (Wright et al. 2015). Cyg OB2 occupies a much larger region than typical young compact star clusters or associations. Based on spatial size of Cyg OB2 and observed age spread, Wright et al. (2015) suggested that Cyg OB2 was not born as a single star cluster but as a 'distribution' of smaller groups or clusters of stars with a range of stellar ages.

According to the H-R diagram, the ages of MT299 and MT317 stars lie in the range between 5 and 6 Myr (see Table 5), while MT259 is younger than $5 \mathrm{Myr}$, and MT282 and MT343 belong to older population of Cyg OB2. In log $g-T_{\text {eff }}$ diagram, MT259 lies in the same area as MT299 and MT317 stars (5-6 Myr), while MT282 and MT343 stars are still in $>10 \mathrm{Myr}$ region. The ages estimated by Wright et al. (2015) are consistent with our estimates within the two standard errors. Thus, comparing our results with results of previous studies we can conclude that the studied stars belong to different subclusters of Cyg OB2: MT259 (B0 V), MT299 (O7.5 V), and MT317 (O8 V) are 4-6 Myr, MT282 (B1 IV) and MT343 (B1 V) are older cluster with 12-14 Myr age.

In addition to the studied stars, in Figures 6 and 7 we marked the A26 (O9.5 V), A33 (B0.2 V), and A38 (O8 V) OB-dwarfs from Negueruela et al. (2008). On the one hand, this shows that the number of studied dwarfs belonging to Cyg OB2 is quite small. Before our work, among the dwarf stars of Cyg OB2 association, only these three stars, as well as MT259 (B0 V) and MT29 (O7 V) studied by Herrero et al. (1999), were modelled. On the other hand, the locations of A26 and A38 together with MT299 and MT317 stars demonstrates the continuity of star-formation process in the association.

\subsection{Mass-loss rate}

O-dwarfs show spectral lines with P-Cyg profiles only in the ultraviolet (UV) range. Therefore, UV spectra are necessary 

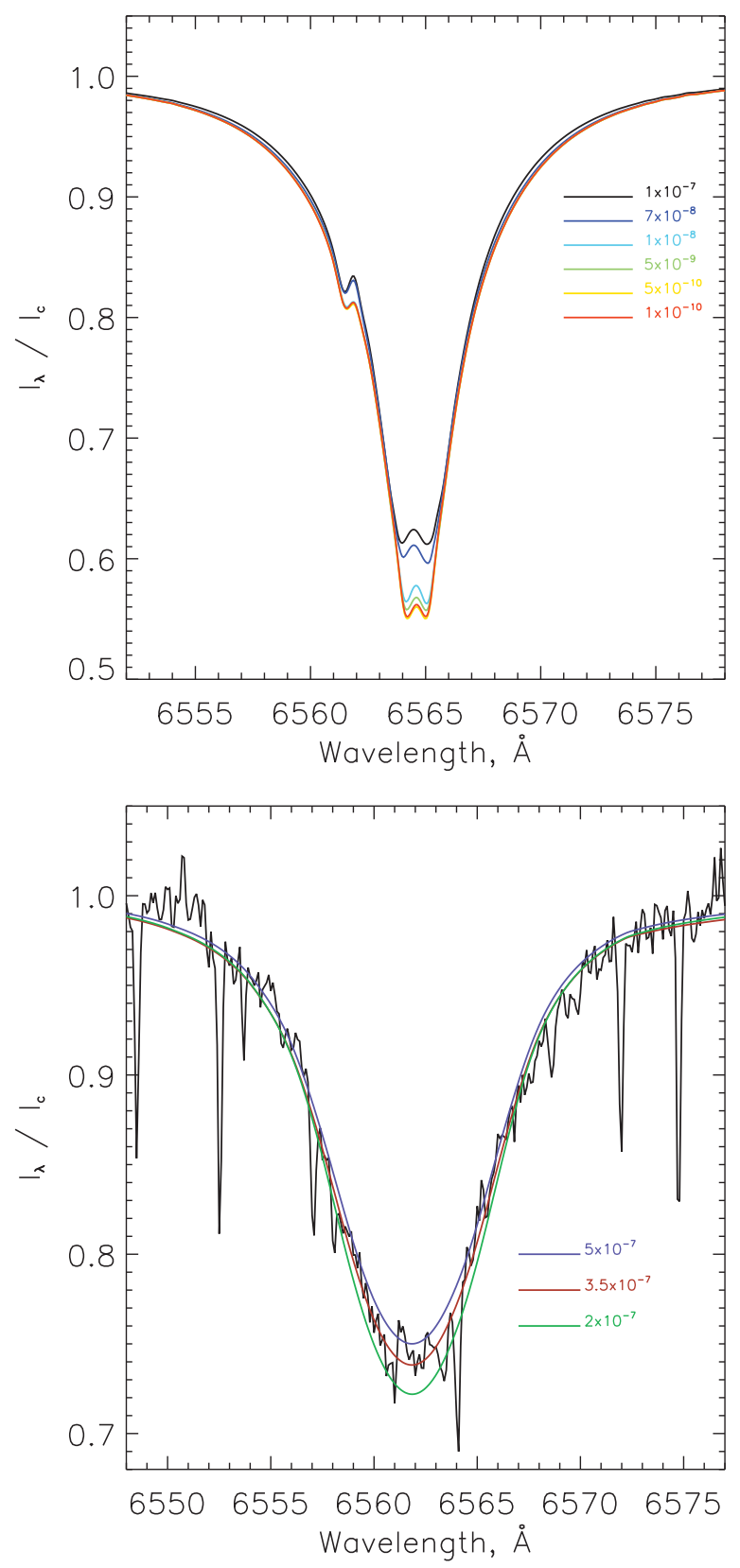

Figure 8. Upper panel: changes of profile of $H_{\alpha}$ line with the decrease of mass loss rate. Model luminosity is $1.2 \cdot 10^{5} \mathrm{~L}_{\odot}$, terminal velocity is $V_{\infty}=1000 \mathrm{~km} \mathrm{~s}^{-1}$. Lower panel: comparison of $H_{\alpha}$ line profile from the spectrum of MT317 (O8 IV) with models computed for different mass loss rates. Mass-loss rate is in units of $\mathrm{M}_{\odot} \mathrm{yr}^{-1}$.

for an accurate assessment of their mass-loss rates. Marcolino (2009) demonstrated that when $\dot{M}$ falls below $10^{-7} \mathrm{M}_{\odot} \mathrm{yr}^{-1}$ the profile of $H_{\alpha}$ line becomes insensitive to its further reduction. Thus, our estimates of $\dot{M}$ obtained based on optical spectra are just upper limits. As an example, the upper panel of Figure 8 demonstrates changes of $H_{\alpha}$ line profile in model spectra with the decrease of mass-loss rate. The lower panel also shows the example of fitting of $H_{\alpha}$ line in the spectum of MT317 (O8 IV) with the model spectra calculated for different values of mass-loss rate.

Tables 5 shows $\dot{M}$ predicted by the theory (Vink, de Koter, \& Lamers 2000, 2001), marked as $\dot{M}_{\text {Vink }}$. The measured mass loss rates are consistent with $\dot{M}_{\text {Vink }}$ predicted.

\section{CONCLUSIONS}

We investigated five stars-MT259 (B0 V), MT282 (B1 IV), MT299 (O7.5 V), MT317 (O8 V), and MT343 (B1 V)belonging to Cyg OB2 association. Using TLUSTY and CMFGEN codes, we estimated their physical parameters, including luminosity, mass-loss rate, projected rotation velocity, and chemical composition of the atmospheres. According to our modelling, we reclassify MT282 and MT317 from dwarfs to subgiants owing to their higher luminosities. $\mathrm{H}-\mathrm{R}$ and $\log g-T_{\text {eff }}$ diagrams show that the ages of MT259, MT299, and MT317 are between 4 and 6 Myr like the ones of most part of Cyg OB2 stars. On the other hand, MT282 and MT343 belong to the older population of the association, their ages $>10$ Myr.

In the paper, we examined in detail the method of automatic comparison of observed spectra with TLUSTY model. We demonstrated that the method works well and it can be used for a simple estimations of stellar parameters of OBstars based on both high- and low-resolution spectra.

We hope that this study will be useful for calibration of the spectral class-luminosity - effective temperature ratio, as the numbers of OB-stars with known distance are still small.

\section{ACKNOWLEDGEMENTS}

The observations at the Russian 6-m telescope were carried out with the financial support of the Ministry of Education and Science of the Russian Federation (agreement No. 14.619.21.0004, project ID RFMEFI61914X0004). We used spectral data retrieved from the ELODIE archive at Observatoire de Haute-Provence (OHP) and database of stellar evolution group at the Geneva Observatory. The study was supported by the Russian Foundation for Basic Research (projects no. 14-02-31247,14-02-00291). Olga Maryeva thanks the grant of Dynasty Foundation. AS was supported by The Ministry of Education and Science of the Russian Federation within the framework of the research activities (project no. 3.1781.2014/K). Also we would like to thank the anonymous referee for valuable comments.

\section{REFERENCES}

Afanasiev, V. L., \& Moiseev, A. V. 2005, AstL, 31, 194

Bouret, J.-C., Lanz, T., Martins, F., Marcolino, W. L. F., Hillier, D. J., Depagne, E., \& Hubeny, I. 2013, A\&A, 555, A1

Bragança, G. A., Daflon, S., Cunha, K., Bensby, T., Oey, M. S.\& Walth, G. 2012, AJ, 144, 130

Castro, N., et al. 2012, A\&A, 542, A79

Chentsov, E. L., Klochkova, V. G., Panchuk, V. E., Yushkin, M. V., \& Nasonov, D. S. 2013, ARep, 57, 527 
Comerón, F., \& Pasquali, A. 2012, A\&A, 543, A101

Comerón, F., et al. 2002, A\&A, 389, 874

Crowther, P. A. 1997, in Proc. IAU Symp. Vol. 189, Fundamental Stellar Properties: the Interaction Between Observation and Theory, eds. T. R. Bedding, A. J. Booth, \& J. Davis (Dordrect: Kluwer), 137

Drew, J. E., Greimel, R., Irwin, M. J., \& Sale, S. E. 2008, MNRAS, 386,1761

Ekström, S., et al. 2012, A\&A, 537, A146

Galazutdinov, G. A. 1996, preprint of the Special Astrophysical Obs. RAS No. 92 (Special Astrophysical Observatory, Nizhnii Arkhyz)

Gies, D. R. 1987, ApJS, 64, 545

Grevesse, N., Asplund, M., \& Sauval, A. J. 2007, SSRv, 130, 105

Guarcello, M. G., Wright, N. J., Drake, J. J., García-Alvarez, D., Drew, J. E., Aldcroft, T., \& Kashyap, V. L. 2012, ApJS, 202, 19

Hanson, M. M. 2003, ApJ, 597, 957

Herrero, A., Corral, L. J., Villamariz, M. R., \& Martín, E. L. 1999, A\&A, 348, 542

Herrero, A., Kudritzki, R. P., Vilchez, J. M., Kunze, D., Butler, K., \& Haser, S. 1992, A\&A, 261, 209

Herrero, A., Puls, J., \& Najarro, F. 2002, A\&A, 396, 949

Hillier, D. J., \& Miller, D. L. 1998, ApJ, 496, 407

Hubeny, I., \& Lanz, T. 1992, A\&A, 262, 501

Hubeny, I., \& Lanz, T. 1995, ApJ, 439, 875

Humphreys, R. M., \& McElroy, D. B. 1984, ApJ, 284, 565

Johnson, H. L., \& Morgan, W. W. 1954, ApJ, 119, 344

Kiminki, D. C., et al. 2007, ApJ, 664, 1102

Kiminki, D. C., Kobulnicky, H. A., Vargas Alvarez, C. A., Alexander, M. J., \& Lundquist, M. J. 2015, ApJ, 811, 85

Knödlseder, J. 2000, A\&A, 360, 539

Kudritzki, R.-P., \& Puls, J. 2000, ARA\&A, 38, 613

Kurucz, R. 1994, Atomic Data for Fe and Ni. Kurucz CDROM No. 22 (Cambridge, MA: Smithsonian Astrophysical Observatory), 22

Lanz, T., \& Hubeny, I. 2003, ApJS, 146, 417

Lanz, T., \& Hubeny, I. 2007, ApJS, 169, 83

Lefever, K., Puls, J., Morel, T., Aerts, C., Decin, L., \& Briquet, M. 2010, A\&A, 515, A74

Marcolino, W. L. F., Bouret, J.-C., Martins, F., Hillier, D. J., Lanz T., \& Escolano, C. 2009, A\&A, 498, 837

Markova, N., \& Puls, J. 2015, in IAU Symposium, 117-118 (arXiv 1409.7784),

Martins, F., Schaerer, D., \& Hillier, D. J. 2005a, A\&A, 436, 1049

Martins, F., Schaerer, D., Hillier, D. J., Meynadier, F., HeydariMalayeri, M., \& Walborn, N. R. 2005b, A\&A, 441, 735

Martins, F., et al. 2015, A\&A, 575, A34

Maryeva, O., Zhuchkov, R., \& Malogolovets, E. 2014, PASA, 31, 20

Maryeva, O. V., Chentsov, E. L., Goranskij, V. P., \& Karpov, S. V. 2016, BaltA

Maryeva, O. V., Klochkova, V. G., \& Chentsov, E. L. 2013, AstBu, 68,87

Massa, D., Fullerton, A. W., Sonneborn, G., \& Hutchings, J. B. 2003, ApJ, 586, 996

Massey, P., \& Thompson, A. B. 1991, ApJ, 101, 1408

Mel'nik, A. M., \& Dambis, A. K. 2009, MNRAS, 400, 518

Morel, T., Hubrig, S., \& Briquet, M. 2008, A\&A, 481, 453

Morgan, W. W., Meinel, A. B., \& Johnson, H. M. 1954, ApJ, 120, 506
Münch, L., \& Morgan, W. W. 1953, ApJ, 118, 161

Najarro, F., Hanson, M. M., \& Puls, J. 2011, A\&A, 535, A32

Negueruela, I., Marco, A., Herrero, A., \& Clark, J. S. 2008, A\&A, 487,575

Panchuk, V. E., Klochkova, V. G., Yushkin, M. V., \& Naidenov, I. D. 2009, JOptT, 76, 87

Panchuk, V. E., Yushkin, M. V., \& Yakopov, M. V. 2011, AstBu, 66, 355

Prugniel, P., \& Soubiran, C. 2001, A\&A, 369, 1048

Przybilla, N., Nieva, M.-F., \& Butler, K. 2008, ApJL, 688, L103

Reddish, V. C., Lawrence, L. C., \& Pratt, N. M. 1966, PROE, 5, 111

Repolust, T., Puls, J., \& Herrero, A. 2004, A\&A, 415, 349

Schulte, D. H. 1956, ApJ, 123, 250

Simón-Díaz, S., \& Herrero, A. 2007, A\&A, 468, 1063

Simón-Díaz, S., \& Herrero, A. 2014, A\&A, 562, A135

Torres-Dodgen, A. V., Carroll, M., \& Tapia, M. 1991, MNRAS, 249,1

Vink, J. S., de Koter, A., \& Lamers, H. J. G. L. M. 2000, A\&A, 362, 295

Vink, J. S., de Koter, A., \& Lamers, H. J. G. L. M. 2001, A\&A, 369, 574

Wright, N. J., Drake, J. J., Drew, J. E., \& Vink, J. S. 2010, ApJ, 713, 871

Wright, N. J., Drew, J. E., \& Mohr-Smith, M. 2015, MNRAS, 449, 741

Wright, N. J., Parker, R. J., Goodwin, S. P., \& Drake, J. J. 2014, MNRAS, 438, 639

Yushkin, M. V., \& Klochkova, V. G. 2005, preptint of the Special Astrophysical Obs. RAS No. 206 (Special Astrophysical Observatory, Nizhnii Arkhyz)

\section{A ESTIMATION OF $\chi_{\mathrm{t}}^{2}$}

The choice of $\chi_{t}^{2}$ given by Equation (2) in Section 3.2 is based on the analysis of spectra of O-stars ALS 8476 (O9 V) and BD+35 1201 (O9.5 V). Spectra of ALS 8476 and BD $+35^{\circ} 1201$ were obtained on the Russian 6-m telescope with the high-resolution NES (Panchuk et al. 2009), spectral resolution is $R=60000$. Continuum normalisation of these spectra had been performed manually with $\mathrm{DECH}$ program (Galazutdinov 1996). The $\mathrm{S} / \mathrm{N}$ of these spectra in the vicinity of HeI $\lambda 5876$ line is 80 and 110 for the ALS 8476 and $\mathrm{BD}+35^{\circ} 1201$ spectra, respectively. But $\mathrm{S} / \mathrm{N}$ is significantly lower $(\sim 30)$ in the blue region of spectra resulting in relatively high parameter errors of ALS 8476 and $\mathrm{BD}+35^{\circ} 1201$.

The projected rotational velocities $v \sin i$ of ALS 8476 and $\mathrm{BD}+35^{\circ} 1201$ were estimated with Full Width at Half Maximum (FWHM) method (see e.g. Herrero et al. 1992). The metal lines were used in the case of ALS 8476 while in the case of BD+35 1201 we used He lines. The obtained values are $v \sin i=18 \pm 4$ and $217 \pm 16 \mathrm{~km} \mathrm{~s}^{-1}$ for ALS 8476 and BD+35 1201 , respectively.

The effective temperature and surface gravity of ALS 8476 and $\mathrm{BD}+35^{\circ} 1201$ were initially estimated by us using the method based on the $T_{\text {eff }}-\log$ g diagnostic diagram [see e.g. Herrero et al. (1992)] with hydrogen and helium lines. The estimated values are $T_{\text {eff }}=32000 \pm 1500 \mathrm{~K}$ and $\log \mathrm{g}=4.1 \pm 0.2 \mathrm{dex}$ for ALS 8476 and $T_{\text {eff }}=32000 \pm 1500 \mathrm{~K}$ and $\log \mathrm{g}=3.9 \pm 0.2 \mathrm{dex}$ for $\mathrm{BD}+35^{\circ} 1201$. Then, the parameters of these two stars were refined using the method for high-resolution spectra described in Section 3.2 with different $\chi_{t}^{2}$ values. The final $\chi_{t}^{2}$ was chosen to provide the values of parameter errors comparable to 
the ones obtained with the method based on the $T_{\text {eff }}-\log g$ diagnostic diagram. With this chosen value of $\chi_{\mathrm{t}}^{2}$, the refined parameters are $T_{\text {eff }}=32000 \pm 1000 \mathrm{~K}$ and $\log \mathrm{g}=4.2 \pm 0.2 \mathrm{dex}$ for ALS 8476 and $T_{\text {eff }}=32500 \pm 1100 \mathrm{~K}$ and $\log \mathrm{g}=4.1 \pm$ 0.2 dex for $\mathrm{BD}+35^{\circ} 1201$. This estimates are consistent with those obtained with $T_{\text {eff }}$-log g diagnostic diagram.

\section{B TESTING THE METHOD OF EFFECTIVE TEMPERATURE AND SURFACE GRAVITY DETERMINATION}

To test the method of automatic spectral analysis described in the Section 3.2, we used spectra of the following stars: HD 15629 [O5 V((f))], HD 34078 (O9.5 V). Moreover, to test the applicability of this method for low-resolution spectra of B-stars we used the spectra of HD 36591 (B1 V) and HD 42597 (B1 V). The parameters of HD 15629, HD 34078, and HD 36591 are known from the literature (Martins et al. 2005b; Lefever et al. 2010) while the spectrum of HD 42597 had not previously been modelled.

The spectra of HD 15629, HD 42597, and HD 36591 were acquired from Elodie archive of Observatoire de HauteProvence (http://atlas.obs-hp.fr/elodie/intro.html) (Prugniel \& Soubiran 2001). The spectrum of HD 34078 obtained on the $1.2-\mathrm{m}$ telescope of the Kourovka Astronomical Observatory of the Ural Federal University with the fiber-fed high-resolution echelle spectrograph (UFES) (Panchuk, Yushkin, \& Yakopov 2011). Continuum normalisation of all spectra had been performed manually with DECH program (Galazutdinov 1996). The spectral resolutions of Elodie and UFES spectra are 42000 and 30000 , correspondingly. $\mathrm{S} / \mathrm{N}$ of Elodie spectra in the vicinity of HeI $\lambda 5876$ line is higher than 100, while for the UFES spectrum it is 75 .

$v \sin i$ values for HD 15629, HD 36591, and HD 42597 were adopted to be the same as in Martins et al. (2005b); SimónDíaz \& Herrero (2014) and Bragança et al. (2012), and equal to 90,9 , and $87 \mathrm{~km} \mathrm{~s}^{-1}$ correspondingly. There are several different estimates of $v \sin i$ for HD 34078-Herrero et al. (1992) give $v \sin i=40 \mathrm{~km} \mathrm{~s}^{-1}$, while Gies (1987) obtained $v \sin i=27 \mathrm{~km} \mathrm{~s}^{-1}$, Martins et al. (2005b) adopted $v \sin i=40 \mathrm{~km} \mathrm{~s}^{-1}$. We obtained $v \sin i=21 \pm 3 \mathrm{~km} \mathrm{~s}^{-1}$ from the Fourier transform of the profiles of metal lines [see e.g. Simón-Díaz \& Herrero (2007)] and used this value in the analysis.

We estimated the parameters of HD 15629 and HD 34078 with method given in Section 3.2 using 9 and 18 spectral lines, correspondingly. We also estimated their parameters with the smaller number of lines which is equal to the number of lines used to estimate parameters of MT259. From Table A1, it is clear that parameters estimated with different number of spectral lines are in good agreement with each other and with the parameters obtained by Martins et al. (2005b) who used TLUSTY and CMFGEN codes. Moreover, we estimated $\mathrm{He} / \mathrm{H}=0.08 \pm 0.02$ for $\mathrm{HD} 15629$ that is in agreement with $\mathrm{He} / \mathrm{H}=0.08$ obtained by Repolust et al. (2004). Figures A1 and A2 show the results of fitting of HD 15629 and HD 34078 observed spectra with the best model.

To test the method for low resolution spectra, we initially estimated $T_{\text {eff }}$ and $\log$ g of HD 42597 and HD 36591 with the procedure described in Section 3.2 and synthetic spectra computed using stellar atmosphere models from the BSTAR2006 (Lanz \& Hubeny 2007). The synthetic spectra were computed with different values of Teff, $\operatorname{logg}, v_{\text {turb }}$, and $\mathrm{He} / \mathrm{H}$. The final values of $T_{\text {eff }}$ and $\log g$ are given in Table A1. The parameters of HD 36591 are well consistent with the estimates of other authors summarised in Table A1. Then, we estimated $T_{\text {eff }}$ and $\log g$ of HD 42597 and HD 36591 using low-resolution spectra of these stars. These low-resolution spectra were obtained from high-resolution spectra by convolving with the instrumental profile with the width corresponding to SCORPIO resolution. We also had added Poisson noise in these low resolution spectra to make their $\mathrm{S} / \mathrm{N}$ comparable to $\mathrm{S} / \mathrm{N}$ of our spectra of MT282 and MT343 stars. Estimates of $T_{\text {eff }}$ and $\log g$ obtained with the same lines and spectral regions as were used for MT343 are presented in Table A1. These estimates are well consistent with those obtained for high resolution spectra. Thus, the method described in Section 3.2 allows to obtain reliable estimates of $T_{\text {eff }}$ and $\log g$ in the case of low spectral resolution. But the parameter errors obtained from low-resolution spectra are relatively high that is mostly due to lower $\mathrm{S} / \mathrm{N}$ of these spectra. These errors are consistent with those that can be estimated comparing the observed and synthetic spectra by eye. Thus, for the analysis of low resolution spectra we used the same value of $\chi_{t}^{2}$ that was found for high-resolution spectra.

Table A1. Testing of the spectral analysis method. The comparison of estimated parameters of HD 15629 [O5 V((f))], HD 34078 (O9.5 V), HD 36591 (B1V), HD 42597 (B1V) with the literature data.

\begin{tabular}{|c|c|c|c|}
\hline Star & $T_{\mathrm{eff}},[\mathrm{kK}]$ & $\log \mathrm{g},[\mathrm{dex}]$ & Source \\
\hline HD 15629 & $40.8 \pm 1.1$ & $3.81 \pm 0.12$ & HeII $\lambda \lambda 4200,4542, \mathrm{HeI} \lambda \lambda 4471,4921,5876$, wings of $\mathrm{H} \delta, \mathrm{H} \gamma, \mathrm{H} \beta, \mathrm{H} \alpha$ \\
\hline \multirow[t]{2}{*}{ O5V ((f)) } & $41.1 \pm 1.1$ & $3.84 \pm 0.12$ & HeII $\lambda 4542$, HeI $\lambda \lambda 4471,4921$, wings of $\mathrm{H} \beta$ \\
\hline & $41 \pm 2.0$ & $3.75 \pm 0.1$ & Martins et al. (2005b) \\
\hline HD 34078 & & & HeII $\lambda \lambda 4200,4542,4686,5411, \mathrm{HeI} \lambda \lambda 4143,4388,4471,4713,4921,5015$ \\
\hline \multirow[t]{3}{*}{$09.5 \mathrm{~V}$} & $33.0 \pm 0.7$ & $4.12 \pm 0.18$ & $\mathrm{HeI} \lambda \lambda 5047,5876,6678,7065$, wings of $\mathrm{H} \delta, \mathrm{H} \gamma, \mathrm{H} \beta, \mathrm{H} \alpha$ \\
\hline & $33.2 \pm 0.7$ & $4.15 \pm 0.18$ & HeII $\lambda 5411, \mathrm{HeI} \lambda \lambda 5876,6678$, wings of $\mathrm{H} \alpha$ \\
\hline & $33.0 \pm 2.0$ & $4.05 \pm 0.1$ & Martins et al. (2005b) \\
\hline HD 42597 & & & Highresolution spectrum. SiII $\lambda \lambda 4128,4130,5056,6347$ \\
\hline \multirow[t]{2}{*}{$\mathrm{B} 1 \mathrm{~V}$} & $22.8 \pm 1.2$ & $3.98 \pm 0.19$ & SiIII $\lambda \lambda 4552,4567,4574,4819,4829,5740$, wings of $\mathrm{H} \delta, \mathrm{H} \gamma, \mathrm{H} \beta, \mathrm{H} \alpha$ \\
\hline & $23.7 \pm 2.8$ & $4.0 \pm 0.3$ & Lowresolution spectrum. Region $\lambda \lambda 4547-4591$, region $\lambda \lambda 4665-4731$ \\
\hline HD 36591 & & & Highresolution spectrum. SiII $\lambda \lambda 4128,4130,5056,6347$ \\
\hline \multirow[t]{5}{*}{$\mathrm{B} 1 \mathrm{~V}$} & $26.1 \pm 0.8$ & $4.15 \pm 0.19$ & SiIII $\lambda \lambda 4552,4567,4574,4819,4829,5740$, wings of $\mathrm{H} \delta, \mathrm{H} \gamma, \mathrm{H} \beta, \mathrm{H} \alpha$ \\
\hline & $26.3 \pm 2.3$ & $4.2 \pm 0.3$ & Low-resolution spectrum. Region $\lambda \lambda 4547-4591$, region $\lambda \lambda 4665-4731$ \\
\hline & $26 \pm 1$ & $3.9 \pm 0.1$ & Lefever et al. (2010) \\
\hline & $27 \pm 1$ & $4.0 \pm 0.2$ & Morel, Hubrig, \& Briquet (2008) \\
\hline & $27 \pm 0.3$ & $4.12 \pm 0.05$ & Przybilla, Nieva, \& Butler (2008) \\
\hline
\end{tabular}



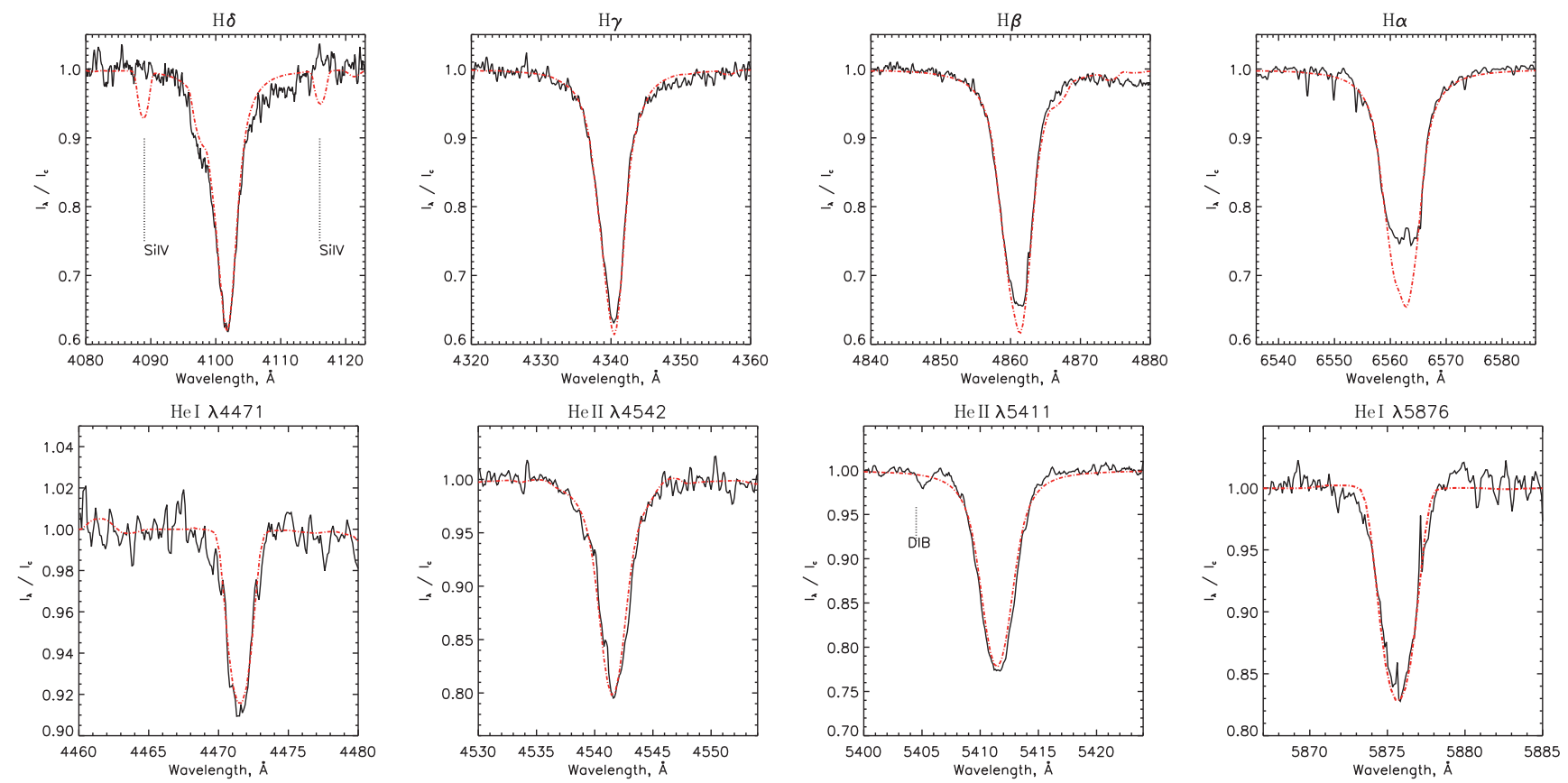

Figure A1. Testing of the spectral analysis method. Modelling of HD 15629 [05 V ((f))]. Comparison of the profiles of selected lines with the best model spectra. The solid line shows the observed profile, and the dashed-dotted line-the TLUSTY-model. The spectrum was obtained with Elodie echelle spectrograph.
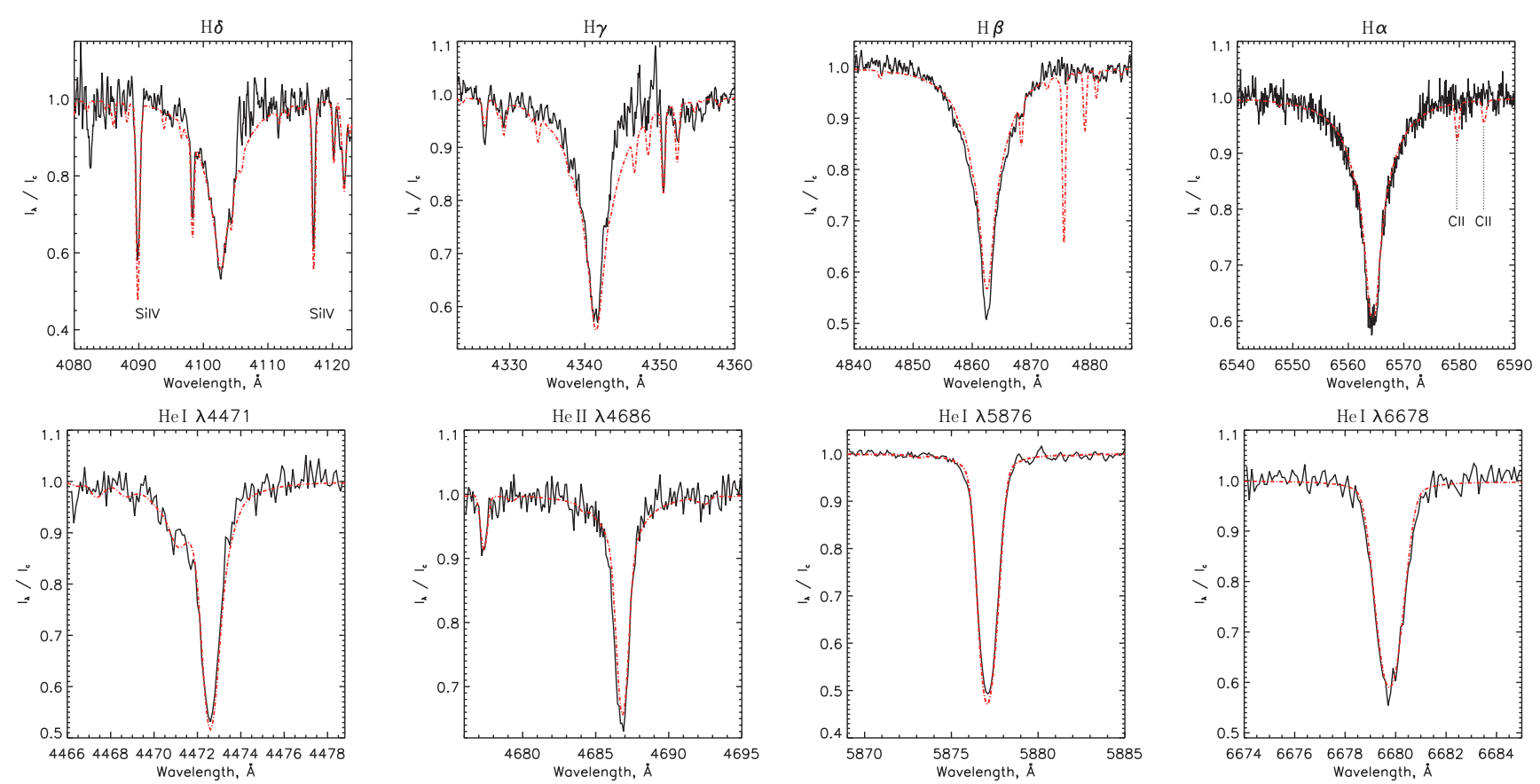

Figure A2. Testing of the spectral analysis method. Modelling of HD 34078 (O9.5 V). Comparison of the profiles of selected lines with the best model spectra. The solid line shows the observed profile, and the dashed-dotted line- the TLUSTY-model. The spectrum was obtained with UFES spectrograph. 